\title{
The Effect of Lipopolysaccharide-containing Moisturizing Cream on Skin Care in Patients With Mild Atopic Dermatitis
}

\author{
KOZO NAKAI $^{1}$, YASUO KUBOTA ${ }^{1}$, GEN-ICHIRO SOMA ${ }^{2}$ and CHIE KOHCHI $^{3}$ \\ ${ }^{1}$ Department of Dermatology, School of Medicine, Kagawa University, Kita-gun, Japan; \\ ${ }^{2}$ Control of Innate Immunity, Technology Research Association, Takamatsu, Japan; \\ ${ }^{3}$ Macrophi Inc., Takamatsu, Japan
}

\begin{abstract}
Background/Aim: Recently, the prevalence of atopic dermatitis $(A D)$ has increased in developed countries. This study aimed to examine the usefulness of a moisturizing cream containing lipopolysaccharide derived from Pantoea agglomerans (LPSp) in patients with mild AD. Patients and Methods: A moisturizing cream containing LPSp or its placebo was randomly assigned and continuously used for 4 weeks in patients with mild $A D$. AD severity was evaluated in a double-blind manner by a dermatologist using the Eczema Area and Severity Index (EASI) score and by the patients' self-evaluation of itching and skin condition using a visual analog scale (VAS). Results: Although there was no difference in the EASI score between the two groups, the VAS scores showed significantly greater symptom alleviation in the LPSP group than in the placebo group. Conclusion: A moisturizing cream containing LPSp may be effective for routine skin care and could help alleviate symptoms of mild $A D$.
\end{abstract}

Atopic dermatitis (AD) is an allergic skin disease with eczematous lesions and severe itching. The prevalence of $\mathrm{AD}$ is estimated to be $15-20 \%$ for children and $1-3 \%$ for adults and has increased 2-3 times in developed countries over the last few decades (1). With this background, daily skin care is very important to avoid worsening of symptoms in patients with $\mathrm{AD}$. The underlying pathology of $\mathrm{AD}$ includes impaired skin barrier function, which is often linked to reduced expression of filaggrin, abnormal proliferation of grampositive bacteria, and an increase in T-helper cell type 2 (Th2) cytokines (2). Therefore, skin care for patients with $\mathrm{AD}$ must involve not only cleanliness and prevention of

This article is freely accessible online.

Correspondence to: Chie Kohchi, Ph.D., Macrophi Inc., DynaxT bldg. 2F, 2217-6, Hayashi-cho, Takamatsu, Kagawa, 761-0301 Japan. Tel: +81878677712, e-mail: kohchi@macrophi.co.jp

Key Words: Skin, itching, LPS, innate immunity. dryness but also desirably assists in increasing the barrier function, suppressing bacterial multiplication, and developing an ideal Th1/Th2 balance.

Gueniche et al. reported that the lysate of a gram-negative bacteria isolated from hot springs is effective as a skin care ingredient for preventing AD aggravation (3). Myles and colleagues also reported that patients with $\mathrm{AD}$ had fewer gram-negative bacteria on the skin than did healthy individuals and that transplanting gram-negative bacteria collected from the skin of healthy individuals to the skin of patients with $\mathrm{AD}$ improved the symptoms of $\mathrm{AD}(4,5)$. Gram-negative bacteria and their lysates contain a large amount of lipopolysaccharide (LPS). LPS is a well-known endotoxin that acts on blood monocytes to induce inflammation (6). LPS is also ubiquitous in the environment and is found in edible plants used as health foods and in traditional Chinese medicine $(7,8)$. It is now clear that LPS is not only associated with no oral percutaneous adverse events, but also induces various beneficial physiological effects, which include natural suppression of the allergic response (9-11).

According to an epidemiological investigation by BraunFahrlander et al., a decline in the natural intake of LPS in early childhood and the onset of allergic disease are inversely correlated (12). There are conflicting reports on this study (13). However, an experimental study that induced AD by applying mite antigen to NC/Nga mice showed that LPS administration reduced serum $\mathrm{IgE}$, periostin, and thymus and activation-regulated chemokine (TARC/CCR7), suppressing atopic symptoms (14). It has also been shown that intradermal administration of LPS inhibits edema in the mouse ear in an immunoglobulin E (IgE)-dependent allergy test system (15). These results suggest that LPS has anti-allergic and antiinflammatory effects. However, the effect of LPS via external application in human AD has not been verified.

Therefore, in order to verify the usefulness of LPS in the remission of patients with mild $\mathrm{AD}$, we evaluated the Eczema Area and Severity Index (EASI) scores (16) and self-evaluation of itching and skin condition, using a visual 
analog scale (VAS), following the use of a moisturizing cream containing LPS derived from Pantoea agglomerans (LPSp), which has been confirmed to be safe by oral and percutaneous application, in a randomized double-blind trial.

\section{Patients and Methods}

Clinical trial and design. This clinical trial was conducted according to the ethical regulations of the World Medical Association (Helsinki Declaration) and was approved by the review committee of the Kagawa University Hospital (approval number: E011CS013). The diagnosis of the skin condition for each trial participant and explanation of the examination to the participant was performed by a dermatologist of the Department of Dermatology, Kagawa University School of Medicine. Informed consent was obtained from all participants before the start of the study, which was conducted as a randomized double-blind parallel group comparison.

Inclusion criteria. Participants satisfied the following criteria: i) men and women between 16 and 65 years of age, ii) a diagnosis of $\mathrm{AD}$ according to a standard guideline (16) by a dermatologist, iii) moderate or mild symptoms (an EASI score $\leq 2$ ) for more than 2 weeks, iv) no regular use of steroids or tacrolimus for 4 weeks prior to the start of the study, and v) written consent to participation in the research.

Diagnosis of $A D$. The symptoms of $\mathrm{AD}$ were diagnosed by a dermatologist. The diagnosis was scored according to the EASI as follows: for each of the four body parts (head and neck, trunk, upper limbs, lower limbs), a numerical value was obtained by evaluating four signs (erythema, papules, scales, and lichenification) at four levels (0: none, 1: mild, 2: moderate, and 3: severe) and adding the scores. These were then multiplied by a numerical value obtained by evaluating the ratio of the area of the eczematous part to that of each body part at seven levels $(0: 0 \%$; 1: $1-9 \%$; $2: 10-29 \% ; 3: 30-49 \%$; $4: 50-69 \% ; 5: 70-89 \%$; and 6: $100 \%$ ) which was then multiplied by the factor for the body part (head and neck: 0.1; trunk: 0.3; upper limb: 0.2; lower limb: 0.4). Finally, obtained numerical values for each of the four body parts were summed up as the EASI score.

VAS analysis. The effectiveness of the cream was investigated by self-evaluation for itching and skin condition using the VAS. The participants rated the severity of itching using a 10-cm VAS from 0 (no itching) to 10 (severe itching) and the skin condition from 0 (no problem at all) to 10 (worst state). The skin conditions were judged comprehensively and visually and included redness, swelling, eczema, dryness, etc.

Moisturizing cream. The placebo control moisturizing cream contained water, olive oil (Croda Japan KK, Tokyo, Japan), glycerin (Miyoshi Oil \& Fat Co., Ltd., Tokyo, Japan), squalane (Nikko Chemicals, Tokyo, Japan), jojoba oil (Nikko Chemicals), butylene glycol (Daicel Corporation, Osaka, Japan), stearic acid (Kao Corporation, Tokyo, Japan), cetearyl alcohol (Kao Corporation), dimethicone (Shin-Etsu Chemical Co., Ltd., Tokyo, Japan), polysorbate 60 (Kao Corporation), glyceryl stearate (SE) (Nikko Chemicals), PEG10 stearate (Nikko Chemicals), carbomer (Fujifilm Wako Pure Chemical Corporation, Tokyo, Japan), tocopherol (Riken Vitamin Co., Ltd., Tokyo, Japan), potassium (Fujifilm Wako Pure
Chemical Corporation), and methylparaben (Ueno Fine Chemicals Industry, Ltd., Osaka, Japan).

The test moisturizing cream containing LPSp was prepared by blending $2 \%$ of cosmetic raw material (INCI name: Pantoea agglomerans/wheat flour ferment extract) (Macrophi Inc., Kagawa, Japan) containing LPSp as the main ingredient into the placebo cream described above. The final concentration of LPSp in the LPSp cream was $2 \mu \mathrm{g} / \mathrm{g}$.

The LPSp cream or placebo was packed in a $30-\mathrm{g}$ capacity plastic container.

Test method. Participants were assigned numbers in the order of registration and then assigned to the LPSp group or placebo group by the central registration center using a randomized table.

Participants received $60 \mathrm{~g}$ (two plastic containers) of LPSp or placebo cream, which was to be applied to the affected area in the morning and evening for 4 weeks. A dermatological diagnosis and self-evaluation were conducted at baseline and at 2 and 4 weeks. Participants filled out self-check sheets every day for the use of their cream and other drugs (such as steroids and tacrolimus). Participants who used steroids or tacrolimus for more than 3 days during the 4-week trial period were excluded from the analysis. Participants who were taking internal medicine (antihistamine) prior to the study continued to take this medicine during the study.

Statistical analysis. Intra-group comparisons were performed using the Wilcoxon signed rank-sum test, and inter-group comparisons were performed using the Mann-Whitney $U$-test. Calculations were made using Excel add-in software for statistical analysis (Excel Statistic 2012; Social Survey Research Information Co., Ltd., Tokyo, Japan). Differences with $p$-values of less than 0.05 were considered statistically significant.

\section{Results}

Participants in this study were males and females with mild AD between the ages of 20 and 52 years. Those who used steroids or tacrolimus for more than 3 days or who discontinued the study during the 4-week trial period were excluded from analysis. The final number of patients to be analyzed was 25 in total, with 12 in the LPSp group (three males, nine females) and 13 in the placebo group (three males, 10 females). The average age for the LPSp group was 31.5 years, whereas that for the placebo group was 31.2 years. The history of AD in all participants analyzed ranged from several years to more than 20 years. The symptoms of AD were most commonly observed in the upper limbs (23 participants), followed by those in the trunk (five participants), lower limbs (four participants), and head (three participants) (Table I).

Figure 1 shows the EASI scores of both groups as evaluated by a dermatologist. In both groups, the EASI scores had improved significantly after 4 weeks of cream application. In the inter-group comparison, there was no statistically significant difference between the two groups at baseline or at 4 weeks; thus, the improvement in EASI scores in both groups was attributed to the moisturizing effect of the creams. 
Table I. Baseline demographics of patients with atopic dermatitis $(A D)$.

\begin{tabular}{|c|c|c|c|c|c|c|c|}
\hline \multirow[t]{2}{*}{ Group } & \multirow[t]{2}{*}{$\mathrm{n}$} & \multirow[t]{2}{*}{ Gender (n) } & \multirow{2}{*}{$\begin{array}{l}\text { Age, mean } \pm \text { SD } \\
\text { (range), years }\end{array}$} & \multirow{2}{*}{$\begin{array}{l}\text { Medical history } \\
\text { of } \mathrm{AD}(\mathrm{n})\end{array}$} & \multirow{2}{*}{$\begin{array}{c}\text { EASI score, } \\
\text { mean } \pm \text { SD (range) }\end{array}$} & \multicolumn{2}{|c|}{ VAS score, mean \pm SD (range) } \\
\hline & & & & & & Skin condition & Itch \\
\hline \multirow[t]{2}{*}{ Placebo } & 13 & M: 3 & $31.5 \pm 6.5(20-52)$ & $\begin{array}{l}15 \text { Years: } 1 \\
\text { 6-10 Years: } 1\end{array}$ & $1.10 \pm 1.18(0.0-4.0)$ & $40.92 \pm 25.83(8-93)$ & $36.42 \pm 30.46(0-83)$ \\
\hline & & F: 10 & & $\begin{array}{l}\text { 11-20 Years: } 3 \\
\geq 21 \text { Years: } 8\end{array}$ & & & \\
\hline LPSp & 12 & $\begin{array}{l}\text { M: } 3 \\
\text { F: } 9\end{array}$ & $31.2 \pm 9.0(22-42)$ & $\begin{array}{l}\text { 6-10 Years: } 1 \\
\text { 11-20 Years: } 3 \\
\geq 21 \text { Years: } 8\end{array}$ & $1.11 \pm 1.34(0.0-4.0)$ & $25.58 \pm 19.00(0-70)$ & $23.83 \pm 23.66(0-75)$ \\
\hline
\end{tabular}

EASI: Eczema area and severity index; F: female; n: number of patients; LPSp: lipopolysaccharide derived from Pantoea agglomerans; M: male; SD: standard deviation; VAS: visual analog scale.

Figure 2 shows the VAS scores for the self-evaluation of skin conditions in both groups. As with the EASI scores, both groups showed improvements with significant differences after 4 weeks. However, the placebo group did not show an improvement beyond 2 weeks. In the intergroup comparison, there was no significant difference between the two groups at the baseline of the study; however, after 4 weeks, the LPSp group showed significantly greater improvement than the placebo group.

Figure 3A shows the VAS scores for the self-evaluation of itching in both groups. In the LPSp group, improvements were observed with significant differences between baseline and at 2 weeks, at 2 and 4 weeks, and at baseline and 4 weeks. However, in the placebo group, although there was a significant improvement at 2 weeks, the improvement stopped from 2 to 4 weeks and no significant difference was observed between scores at baseline and at 4 weeks. In the inter-group comparison, there was no significant difference between the two groups at baseline, but after 4 weeks, the LPSp group showed significantly greater improvement than the placebo group.

Figure $3 \mathrm{~B}$ is a plot of the self-evaluation of itching in all patients, which shows a gradual improvement in almost all patients in the LPSp group throughout the 4 weeks. In contrast, there were many patients from the placebo group in whom itching had worsened from 2 to 4 weeks. This suggests that the LPSp component of the cream had an itchsuppressing effect.

\section{Discussion}

In this randomized double-blind trial on the daily usefulness of an LPSp-blended moisturizing cream in patients with mild $\mathrm{AD}$, improvements were observed in three evaluation items in both the treatment and placebo groups within 4 weeks. This indicates that the moisturizing cream itself had a beneficial effect on the improvement in patients with mild
AD. In the inter-group comparison, no difference was found in the EASI scores, but there was a strong tendency for greater alleviation with the LPSp cream according to the self-evaluation scores for itching and skin conditions.

The LPS used in this study was derived from P. agglomerans, which coexists with edible plants. It has been reported that various diseases are improved by oral or transdermal administration of LPSp (11) and, since 2007, raw materials for food and cosmetics containing LPSp as an active ingredient have been marketed. The chemical structure of LPSp has been well researched: the number of fatty acid residues in Iipid A, an active center of LPS, is 6-7 (17), the sugars in the $O$ antigen polysaccharide are rhamnose and glucose (18), and the molecular weight is 5000-20000 Da (19). Like LPS derived from Escherichia coli, LPSp binds to toll-like receptor 4 (TLR4) in order to exert its biological activity. Normally, substances the size of LPS do not penetrate the tight junction of the granular layer of the epidermis (20). However, the lipid feature of the LPSp molecule seems to confer an affinity for the stratum corneum of the epidermis in which sebum exists, allowing interaction with cells in the stratum corneum.

It was notable that the tendency for self-evaluated improvement in itching was more remarkable in the LPSp group than in the placebo group in our study. Itching significantly affects the quality of life of patients with AD and further worsens AD by destroying the skin's barrier function. Itching in $\mathrm{AD}$ has been a focus of recent attention with research on the expression of interleukin 31 (IL31) by Th2 cells (21), nerve activation by thymic stromal lymphopoietin (TSLP) (22), and the increase in number of nerve fibers related to the relative expression state between nerve growth factor and the nerve repulsion factor semaphorin 3A $(23,24)$. The relationship between these phenomena and LPS has not yet been investigated, but there are some relevant findings.

Although regulatory T-cells (Tregs), which suppress helper T-cells, exist in the epidermis of a healthy person, the 


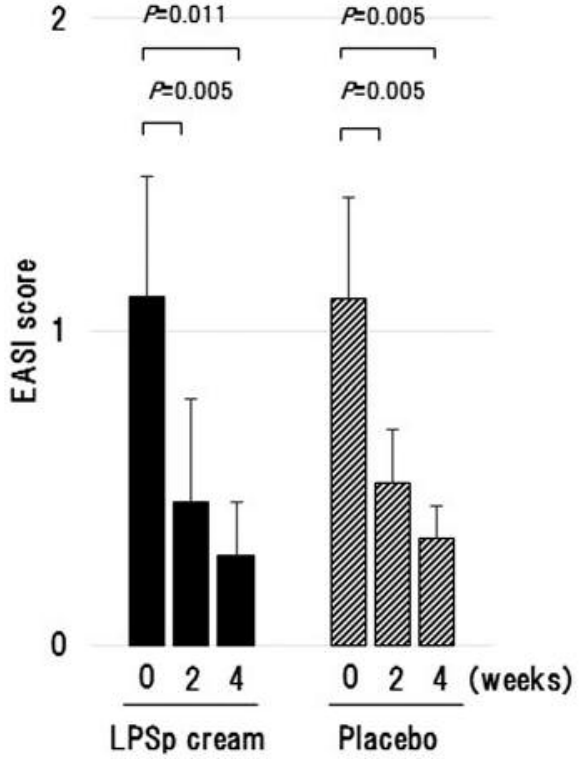

Figure 1. Eczema Area and Severity Index (EASI) scores in the lipopolysaccharide derived from Pantoea agglomerans (LPSp) and placebo groups. Average EASI score, as evaluated by a dermatologist over time (0; baseline, 2 weeks, 4 weeks). Data are presented as the mean \pm standard error (SE). Statistical analysis was performed using the Wilcoxon signed rank-sum test (intra-group comparison) and MannWhitney U-test (inter-group comparison). Significance is shown only where a statistically significant $(p<0.05)$ difference was found.

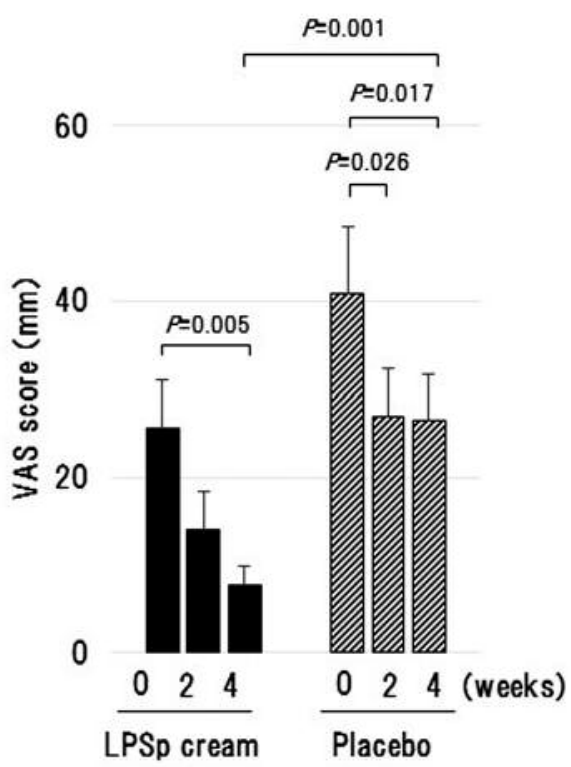

Figure 2. Visual analog scores (VAS) for the self-evaluation of skin condition in the lipopolysaccharide derived from Pantoea agglomerans (LPSp) and placebo groups. Average VAS scores for skin condition were self-evaluated over time (0, baseline; 2 weeks; 4 weeks). Data are presented as mean \pm standard error (SE). Statistical analysis was performed using the Wilcoxon signed rank-sum test (intra-group comparison) and Mann-Whitney U-test (inter-group comparison). Significance is shown only where a statistically significant $(p<0.05)$ difference was found.
A

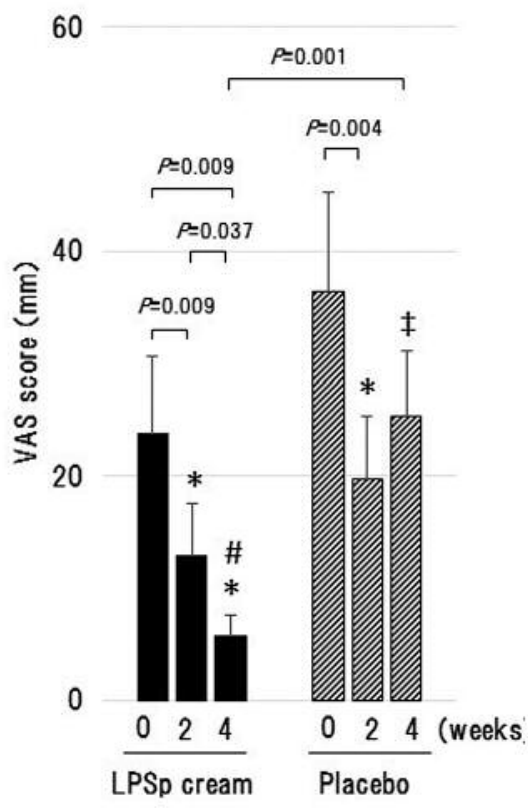

B

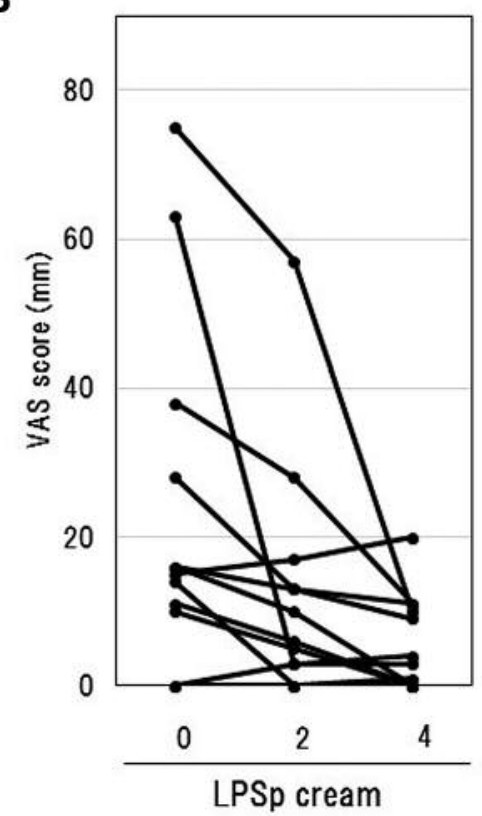

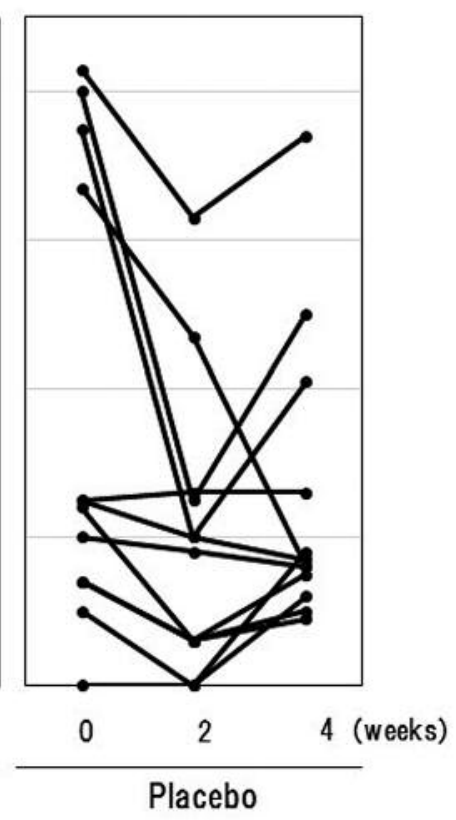

Figure 3. Visual analog scores (VAS) for self-evaluation of itching in the lipopolysaccharide derived from Pantoea agglomerans (LPSp) and placebo groups. A: Average VAS values for itching, as self-evaluated over time. Data are presented as mean \pm standard error (SE). Statistical analysis was performed using the Wilcoxon signed rank-sum test (intra-group comparison) and Mann-Whitney U-test (inter-group comparison). B: Individual VAS values for itching as self-evaluated over time in the LPSp group (left) and the placebo group (right). Significantly different at $p<0.05$ versus *corresponding baseline value (0 weeks), ${ }^{*}$ corresponding value at 2 weeks, ${ }^{*}$ corresponding LPSp value. 
function of Tregs is thought to be depressed in patients with AD $(25,26)$. On the other hand, Tregs express TLR4 and respond to LPS in survival and proliferation (27). It has also been reported that LPS-stimulated Tregs affect neutrophils in an immunosuppressive manner, so that they suppress inflammation $(28,29)$. From this, it is also conceivable that LPS activates Tregs to suppress helper T-cells and reduce the expression of IL31, which causes inflammation and itching.

Although many of the interactions between LPS and other cells present in the epidermis are still not understood, there have been some elucidating reports. Keratinocytes express TLRs, including TLR4 but not TLR7 and TLR8, and react to LPS stimulation. The induction of inflammatory cytokines in keratinocytes is lower (30) compared with that in monocytes in the blood. However, Isard et al. showed that stimulation of keratinocytes with LPS increases the expression level of filaggrin, a barrier function protein (31). In addition, keratinocytes produce $\beta$-defensin, an antimicrobial substance, in vivo in response to stimulation by LPS (32). From these findings, LPS may act on keratinocytes to enhance the skin's barrier function and suppress abnormal bacterial multiplication.

Langerhans cells protrude dendrites into the stratum corneum (33). In Langerhans cells, there is no induction of inflammatory cytokines in response to LPS stimulation (34). Rather, LPS stimulation suppresses the expression of Th2 chemokine TARC (35). This suggests that the action of LPS on Langerhans cells leads to suppression of the allergic response.

In the present study, it was shown that moisturizing cream, a basic cosmetic, was useful in the daily skin care of patients with mild AD. Furthermore, the results suggested that blending LPS into moisturizing cream is effective for improving itching. LPS, a lipoglycan peculiar to gram-negative bacteria, is also an endotoxin and induces strong inflammation when brought into the body by infection or by artificial injection. However, LPS is ubiquitous in the environment, including edible plants and herbal medicines used for health foods. Because toxicity from oral and dermal ingestion of LPS is not observed, it is obvious that physiological response to LPS by the cells of the intestinal tract and skin that are in contact with the environment and resident bacteria is quite different from that by immune cells in the body.

This clinical trial was conducted as the first test in humans to verify the usefulness of LPS; however, the sample size was small. In the future, clinical trials with larger sample sizes are necessary. In addition, in order to clarify the mechanism of the effect, it will be necessary to study the interaction between LPS and skin cells.

\section{Conflicts of Interest}

The Authors declare that no conflict of interest exists in regard to this research.

\section{Acknowledgements}

The Authors would like to thank the patients who participated for their cooperation in this study. Additionally, we would like to thank Dr. Hiroyuki Inagawa and Yoko Nakata for cooperation and advice on statistical analysis. The Authors would like to thank Enago (www.enago.jp) for the English language review.

\section{References}

1 Nutten S: Atopic dermatitis: Global epidemiology and risk factors. Ann Nutr Metab 66(Suppl 1): 8-16, 2015.

2 Peng W and Novak N: Pathogenesis of atopic dermatitis. Clin Exp Allergy 45(3): 566-574, 2015.

3 Gueniche A, Knaudt B, Schuck E, Volz T, Bastien P, Martin R, Rocken $\mathrm{M}$, Breton $\mathrm{L}$ and Biedermann $\mathrm{T}$ : Effects of nonpathogenic gram-negative bacterium Vitreoscilla filiformis lysate on atopic dermatitis: A prospective, randomized, doubleblind, placebo-controlled clinical study. Br J Dermatol 159(6): 1357-1363, 2008.

4 Myles IA, Williams KW, Reckhow JD, Jammeh ML, Pincus NB, Sastalla I, Saleem D, Stone KD and Datta SK: Transplantation of human skin microbiota in models of atopic dermatitis. JCI Insight 1(10): e86955, 2016.

5 Myles IA, Earland NJ, Anderson ED, Moore IN, Kieh MD, Williams KW, Saleem A, Fontecilla NM, Welch PA, Darnell DA, Barnhart LA, Sun AA, Uzel G and Datta SK: First-in-human topical microbiome transplantation with Roseomonas mисоsa for atopic dermatitis. JCI Insight 3(9): e120608, 2018.

6 Kulp A and Kuehn MJ: Biological functions and biogenesis of secreted bacterial outer membrane vesicles. Annu Rev Microbiol 64: 163-184, 2010.

7 Inagawa H, Nishizawa T, Tsukioka D, Suda T, Chiba Y, Okutomi T, Morikawa A, Soma GI and Mizuno D: Homeostasis as regulated by activated macrophage. II. LPS of plant origin other than wheat flour and their concomitant bacteria. Chem Pharm Bull 40(4): 994-997, 1992.

8 Montenegro D, Kalpana K, Chrissian C, Sharma A, Takaoka A, Iacovidou M, Soll CE, Aminova O, Heguy A, Cohen L, Shen S and Kawamura A: Uncovering potential 'herbal probiotics' in Juzen-taiho-to through the study of associated bacterial populations. Bioorg Med Chem Lett 25(3): 466-469, 2015.

9 Brandl K, Plitas G, Mihu CN, Ubeda C, Jia T, Fleisher M, Schnabl B, DeMatteo RP and Pamer EG: Vancomycin-resistant enterococci exploit antibiotic-induced innate immune deficits. Nature 455(7214): 804-807, 2008.

10 Chen LW, Chang WJ, Chen PH and Hsu CM: Commensal microflora induce host defense and decrease bacterial translocation in burn mice through toll-like receptor 4. J Biomed Sci 17: 48, 2010

11 Kohchi C, Inagawa H, Nishizawa T, Yamaguchi T, Nagai S and Soma G: Applications of lipopolysaccharide derived from Pantoea agglomerans (IP-PA1) for health care based on macrophage network theory. J Biosci Bioeng 102(6): 485-496, 2006.

12 Braun-Fahrlander C, Riedler J, Herz U, Eder W, Waser M, Grize L, Maisch S, Carr D, Gerlach F, Bufe A, Lauener RP, Schierl R, Renz H, Nowak D, von Mutius E, Allergy and Endotoxin Study T: Environmental exposure to endotoxin and its relation to asthma in school-age children. N Engl J Med 347(12): 869-877, 2002. 
13 Flohr C and Yeo L: Atopic dermatitis and the hygiene hypothesis revisited. Curr Probl Dermatol 41: 1-34, 2011.

14 Wakame $\mathrm{K}$, Komatsu $\mathrm{K}$, Inagawa $\mathrm{H}$ and Nishizawa $\mathrm{T}$ : Immunopotentiator from Pantoea agglomerans prevents atopic dermatitis induced by dermatophagoides farinae extract in NC/Nga mouse. Anticancer Res 35(8): 4501-4508, 2015.

15 Inagawa H, Nishizawa T, Takagi K, Mizuno D and Soma G-I: Protective effect by intradermal administration of Pantoea agglomerans LPS (LPSp) and oral administration of ONO-4007, a lipid A derivative, on IgE-dependent ear swelling. Biotherapy 11(3): 464-466, 1997.

16 Furue M, Saeki, H, Frukawa F, Hide M, Ohtsuki M, Katayama I, Sasaki R, Suto H and Takehara, K: Guidelines for management of atopic dermatitis. J Dermatol 119(8): 1515-1534, 2009.

17 Tsukioka D, Nishizawa T, Miyase T, Achiwa K, Suda T, Soma G and Mizuno D: Structural characterization of lipid A obtained from Pantoea agglomerans lipopolysaccharide. FEMS Microbiol Lett 149(2): 239-244, 1997.

18 Hashimoto M, Satou R, Ozono M, Inagawa H and Soma GI: Characterization of the $\mathrm{O}$-antigen polysaccharide derived from Pantoea agglomerans IG1 lipopolysaccharide. Carbohydr Res 449: 32-36, 2017.

19 Nishizawa T, Inagawa H, Oshima H, Okutomi T, Tsukioka D, Iguchi M, Soma G and Mizuno D: Homeostasis as regulated by activated macrophage. I. Lipopolysaccharide (LPS) from wheat flour: Isolation, purification and some biological activities. Chem Pharm Bull 40(2): 479-483, 1992.

20 Furuse M, Hata M, Furuse K, Yoshida Y, Haratake A, Sugitani Y, Noda T, Kubo A and Tsukita S: Claudin-based tight junctions are crucial for the mammalian epidermal barrier: A lesson from claudin-1-deficient mice. J Cell Biol 156(6): 1099-1111, 2002.

21 Sonkoly E, Muller A, Lauerma AI, Pivarcsi A, Soto H, Kemeny L, Alenius H, Dieu-Nosjean MC, Meller S, Rieker J, Steinhoff M, Hoffmann TK, Ruzicka T, Zlotnik A and Homey B: IL-31: A new link between T-cells and pruritus in atopic skin inflammation. J Allergy Clin Immunol 117(2): 411-417, 2006.

22 Wilson SR, The L, Batia LM, Beattie K, Katibah GE, McClain SP, Pellegrino M, Estandian DM and Bautista DM: The epithelial cell-derived atopic dermatitis cytokine TSLP activates neurons to induce itch. Cell 155(2): 285-295, 2013.

23 Tominaga M, Ogawa $\mathrm{H}$ and Takamori K: Decreased production of semaphorin $3 \mathrm{~A}$ in the lesional skin of atopic dermatitis. $\mathrm{Br} \mathrm{J}$ Dermatol 158(4): 842-844, 2008.

24 Tominaga $\mathrm{M}$ and Takamori $\mathrm{K}$ : Itch and nerve fibers with special reference to atopic dermatitis: Therapeutic implications. J Dermatol 41(3): 205-212, 2014.

$25 \mathrm{Ou}$ LS, Goleva E, Hall C and Leung DY: T-Regulatory cells in atopic dermatitis and subversion of their activity by superantigens. J Allergy Clin Immunol 113(4): 756-763, 2004.
26 Verhagen J, Akdis M, Traidl-Hoffmann C, Schmid-Grendelmeier $\mathrm{P}$, Hijnen D, Knol EF, Behrendt H, Blaser K and Akdis CA: Absence of T-regulatory cell expression and function in atopic dermatitis skin. J Allergy Clin Immunol 117(1): 176-183, 2006.

27 Caramalho I, Lopes-Carvalho T, Ostler D, Zelenay S, Haury M and Demengeot J: Regulatory T-cells selectively express toll-like receptors and are activated by lipopolysaccharide. J Exp Med 197(4): 403-411, 2003.

28 Lewkowicz N, Klink M, Mycko MP and Lewkowicz P: Neutrophil-CD4+CD25+ T-regulatory cell interactions: A possible new mechanism of infectious tolerance. Immunobiology 218(4): 455-464, 2013.

29 Lewkowicz P, Lewkowicz N, Sasiak A and Tchorzewski H: Lipopolysaccharide-activated $\mathrm{CD} 4{ }^{+} \mathrm{CD} 25^{+}$T-regulatory cells inhibit neutrophil function and promote their apoptosis and death. J Immunol 177(10): 7155-7163, 2006.

30 Lebre MC, van der Aar AM, van Baarsen L, van Capel TM, Schuitemaker JH, Kapsenberg ML and de Jong EC: Human keratinocytes express functional Toll-like receptor 3, 4, 5, and 9. J Invest Dermatol 127(2): 331-341, 2007.

31 Isard O, Knol AC, Aries MF, Nguyen JM, Khammari A, CastexRizzi N and Dreno B: Propionibacterium acnes activates the IGF-1/IGF-1R system in the epidermis and induces keratinocyte proliferation. J Invest Dermatol 131(1): 59-66, 2011.

32 Seo SJ, Ahn SW, Hong CK and Ro BI: Expressions of betadefensins in human keratinocyte cell lines. J Dermatol Sci 27(3): 183-191, 2001

33 Kubo A, Nagao K, Yokouchi M, Sasaki H and Amagai M: External antigen uptake by Langerhans cells with reorganization of epidermal tight junction barriers. J Exp Med 206(13): 29372946, 2009.

34 van der Aar AM, Sylva-Steenland RM, Bos JD, Kapsenberg ML, de Jong EC and Teunissen MB: Loss of TLR2, TLR4, and TLR5 on Langerhans cells abolishes bacterial recognition. J Immunol 178(4): 1986-1990, 2007.

35 Mitsui H, Watanabe T, Saeki H, Mori K, Fujita H, Tada Y, Asahina A, Nakamura K and Tamaki K: Differential expression and function of Toll-like receptors in Langerhans cells: Comparison with splenic dendritic cells. J Invest Dermatol 122(1): 95-102, 2004. 